\title{
A NOTE ON FINITE DUAL FRAME PAIRS
}

\author{
OLE CHRISTENSEN, ALEXANDER M. POWELL, AND XIANG CHUN XIAO
}

(Communicated by Michael T. Lacey)

\begin{abstract}
The purpose of this note is to extend certain key results in frame theory from the setting of tight frames to dual pairs of frames. We provide various characterizations of dual frame pairs $\left\{e_{n}\right\}_{n=1}^{N},\left\{f_{n}\right\}_{n=1}^{N}$ for a $d$-dimensional Hilbert space $\mathbb{K}^{d}$. Based on this we characterize those scalar sequences $\left\{\alpha_{n}\right\}_{n=1}^{N}$ for which there exist dual pairs of frames $\left\{e_{n}\right\}_{n=1}^{N},\left\{f_{n}\right\}_{n=1}^{N}$ for $\mathbb{K}^{d}$ such that $\alpha_{n}=\left\langle e_{n}, f_{n}\right\rangle$. This generalizes the well-known fundamental inequality of tight frames.
\end{abstract}

\section{INTRODUCTION}

Frames in finite-dimensional spaces have been studied intensively during the past decade as tools for signal and data representation; see [1, 2, 5, 10. Due to their computational simplicity and robustness features, one direction of focus has been on the construction of tight frames in $\mathbb{R}^{d}$ and $\mathbb{C}^{d}$; see, e.g., [2, 7, 8]. However, it is also known that general frame pairs can sometimes provide increased flexibility and performance benefits over tight frames; e.g., see [3]. The purpose of this note is to extend certain key results from the setting of tight frames to dual pairs of frames. We provide various characterizations of dual frame pairs $\left\{e_{n}\right\}_{n=1}^{N},\left\{f_{n}\right\}_{n=1}^{N}$ for a $d$-dimensional Hilbert space. Based on this we characterize those scalar sequences $\left\{\alpha_{n}\right\}_{n=1}^{N}$ for which there exist dual pairs of frames $\left\{e_{n}\right\}_{n=1}^{N},\left\{f_{n}\right\}_{n=1}^{N}$ such that $\alpha_{n}=\left\langle e_{n}, f_{n}\right\rangle$. This generalizes the well-known fundamental inequality of tight frames.

Throughout this note $\mathbb{K}^{d}$ denotes either $\mathbb{R}^{d}$ or $\mathbb{C}^{d}$. Recall that a sequence $\left\{e_{n}\right\}_{n=1}^{N} \subset \mathbb{K}^{d}$ is a frame for $\mathbb{K}^{d}$ with frame bounds $A, B>0$ if

$$
\forall x \in \mathbb{K}^{d}, \quad A\|x\|^{2} \leq \sum_{n=1}^{N}\left|\left\langle x, e_{n}\right\rangle\right|^{2} \leq B\|x\|^{2} .
$$

Note that $\left\{e_{n}\right\}_{n=1}^{N} \subset \mathbb{K}^{d}$ is a frame if and only if $\operatorname{span}\left\{e_{n}\right\}_{n=1}^{N}=\mathbb{K}^{d}$.

Given a frame $\left\{e_{n}\right\}_{n=1}^{N} \subset \mathbb{K}^{d}$ it is well known that there exists at least one sequence $\left\{f_{n}\right\}_{n=1}^{N} \subset \mathbb{K}^{d}$ for which the frame expansions

$$
\forall x \in \mathbb{K}^{d}, \quad x=\sum_{n=1}^{N}\left\langle x, e_{n}\right\rangle f_{n}=\sum_{n=1}^{N}\left\langle x, f_{n}\right\rangle e_{n}
$$

hold. Any such sequence $\left\{f_{n}\right\}_{n=1}^{N}$ is called a dual frame, and the systems $\left\{e_{n}\right\}_{n=1}^{N}$ and $\left\{f_{n}\right\}_{n=1}^{N}$ are referred to as a dual frame pair. If $E$ and $F$ are the $d \times N$ matrices whose $n$th columns are $e_{n}$ and $f_{n}$ respectively, then (1.2) is equivalent to

Received by the editors January 7, 2011 and, in revised form, May 10, 2011.

2010 Mathematics Subject Classification. Primary 42C15. 
$F E^{*}=E F^{*}=I_{d \times d}$. The matrices $E, F$ are referred to as the synthesis operators associated to $\left\{e_{n}\right\}_{n=1}^{N},\left\{f_{n}\right\}_{n=1}^{N}$, whereas $E^{*}, F^{*}$ are the analysis operators. We use $E^{*}$ to denote the adjoint (transpose conjugate) of a matrix and use $E^{T}$ to simply denote the transpose.

When $N>d$ the choice of a dual frame is not unique, but there is an important canonical choice. Consider the frame operator $S: \mathbb{K}^{d} \rightarrow \mathbb{K}^{d}$ defined by $S x=E E^{*} x=\sum_{n=1}^{N}\left\langle x, e_{n}\right\rangle e_{n}$. The frame operator $S$ is self-adjoint, and all of its eigenvalues belong to the interval $[A, B]$. The canonical dual frame $\left\{\widetilde{e}_{n}\right\}_{n=1}^{N}$ associated to $\left\{e_{n}\right\}_{n=1}^{N}$ is defined by $\widetilde{e}_{n}=S^{-1} e_{n}$. It can be verified that $\left\{\widetilde{e}_{n}\right\}_{n=1}^{N}$ is indeed a dual frame. However, there are settings where noncanonical (alternative) dual frames perform better than the canonical dual. For example, in Sigma-Delta quantization of finite frame expansions, the best approximation-theoretic properties are obtained using noncanonical dual frames [12, 3, 13. Other examples related to the uncertainty principle show that noncanonical dual frames can provide improved time-frequency localization over canonical dual frames; see [11.

A frame $\left\{e_{n}\right\}_{n=1}^{N} \subset \mathbb{K}^{d}$ is said to be tight if we can take $A=B$ in (1.1). For tight frames the canonical dual frame is given by $\widetilde{e}_{n}=\frac{1}{A} e_{n}$ and the frame expansion (1.2) simplifies to

$$
\forall x \in \mathbb{K}^{d}, \quad x=\frac{1}{A} \sum_{n=1}^{N}\left\langle x, e_{n}\right\rangle e_{n} .
$$

The simplicity of (1.3) makes tight frames especially desirable in practice. Moreover, tight frames are known to be optimally robust against noise in certain settings; see [10. In view of this, characterizations and constructions of tight frames have attracted substantial attention. For a general overview of frames, including tight frames, see [9].

An important early insight 2] into tight frames provides a physical interpretation in terms of minimal energy configurations. As a part of this program the following theorem characterizes the possible norms $\left\|e_{n}\right\|$ of the elements in tight frames; see [4]. The inequality in condition (2) below is known as the fundamental inequality of tight frames.

Theorem 1.1. Given $\left\{a_{n}\right\}_{n=1}^{N} \subset[0, \infty)$ and $N \geq d$, the following are equivalent:

(1) There exists a tight frame $\left\{e_{n}\right\}_{n=1}^{N}$ for $\mathbb{K}^{d}$ such that $a_{n}=\left\langle e_{n}, e_{n}\right\rangle$ for all $1 \leq n \leq N$.

(2) $\max \left\{a_{n}\right\}_{n=1}^{N} \leq \frac{1}{d} \sum_{n=1}^{N} a_{n}$.

Overview and main results. The main goal of this note is to extend Theorem 1.1 to the setting of dual frame pairs. In Section 2 we present various characterizations of dual frame pairs. In Section 3 we show that if $N>d$ and $\left\{\alpha_{n}\right\}_{n=1}^{N} \subset \mathbb{K}$ is a given sequence, then there exists a dual frame pair $\left\{e_{n}\right\}_{n=1}^{N},\left\{f_{n}\right\}_{n=1}^{N}$ for $\mathbb{K}^{d}$ with $\alpha_{n}=\left\langle e_{n}, f_{n}\right\rangle$ if and only if $d=\sum_{n=1}^{N} \alpha_{n}$ (see Theorem 3.1). Surprisingly, for any given $N, d \in \mathbb{N}$ with $N>d$ one can find a frame $\left\{e_{n}\right\}_{n=1}^{N}$ such that the possible dual pair inner products $\left\{\left\langle e_{n}, f_{n}\right\rangle\right\}_{n=1}^{N}$ assume the full possible range described by this condition. We characterize such frames and exhibit a particularly simple choice (see Theorem 3.4 and Example 3.8). Finally, the technical proofs of the results in Section 3 are collected in Section 4 . 


\section{Characterizations of dual frame pairs}

The following proposition provides various characterizations of dual frame pairs. For given sequences $\left\{e_{n}\right\}_{n=1}^{N},\left\{f_{n}\right\}_{n=1}^{N}$ in $\mathbb{K}^{d}$ define the $N \times N$ cross-Gramian and Gramian matrices $\mathcal{A}, \mathcal{B}, \mathcal{C}$ by their $(i, j)$ th entries:

$$
\mathcal{A}_{i j}=\left\langle e_{i}, f_{j}\right\rangle, \quad \mathcal{B}_{i j}=\left\langle e_{i}, e_{j}\right\rangle, \quad \mathcal{C}_{i j}=\left\langle f_{i}, f_{j}\right\rangle .
$$

Proposition 2.1. Let $\left\{e_{n}\right\}_{n=1}^{N}$ and $\left\{f_{n}\right\}_{n=1}^{N}$ be frames for $\mathbb{K}^{d}$. Let $\left\{\widetilde{e}_{n}\right\}_{n=1}^{N}$ denote the canonical dual frame of $\left\{e_{n}\right\}_{n=1}^{N}$. The following are equivalent:

(1) $\left\{e_{n}\right\}_{n=1}^{N}$ and $\left\{f_{n}\right\}_{n=1}^{N}$ are a dual frame pair for $\mathbb{K}^{d}$.

(2) There exists $\left\{z_{n}\right\}_{n=1}^{N} \subset \mathbb{K}^{d}$ such that $f_{n}=\widetilde{e}_{n}+z_{n}$ and

$$
\forall x \in \mathbb{K}^{d}, \quad 0=\sum_{n=1}^{N}\left\langle x, z_{n}\right\rangle e_{n}=\sum_{n=1}^{N}\left\langle x, e_{n}\right\rangle z_{n} .
$$

(3) $\mathcal{A}=\mathcal{B C}$.

(4) $\mathcal{A}^{2}=\mathcal{A}$.

(5) For each $1 \leq n \leq d$ the column vector $\left[\left\langle e_{1}, f_{n}\right\rangle,\left\langle e_{2}, f_{n}\right\rangle, \ldots,\left\langle e_{d}, f_{n}\right\rangle\right]^{T}$ is an eigenvector of $\mathcal{A}$ with eigenvalue 1 .

(6) $\forall 1 \leq k, l \leq d, \quad \sum_{n=1}^{N} e_{j l} \overline{f_{j k}}=\delta_{k, l}$.

Proof. The equivalence $(1) \Leftrightarrow(2)$ is well known; for example, see [14. The equivalence $(4) \Leftrightarrow(5)$ follows from the definition of $\mathcal{A}$.

$(1) \Leftrightarrow(3)$. Note that

$$
(\mathcal{B C})_{i k}=\sum_{j=1}^{N} \mathcal{B}_{i j} \mathcal{C}_{j k}=\sum_{j=1}^{N}\left\langle e_{i}, e_{j}\right\rangle\left\langle f_{j}, f_{k}\right\rangle=\left\langle\sum_{j=1}^{N}\left\langle e_{i}, e_{j}\right\rangle f_{j}, f_{k}\right\rangle .
$$

Because $\operatorname{span}\left\{f_{n}\right\}_{n=1}^{N}=\mathbb{K}^{d}$ this shows that $\mathcal{A}=\mathcal{B C}$ if and only if $e_{i}=\sum_{j=1}^{N}\left\langle e_{i}, e_{j}\right\rangle f_{j}$ holds for $i=1, \ldots, N$. Because $\operatorname{span}\left\{e_{n}\right\}_{n=1}^{N}=\mathbb{K}^{d}$, this in turn holds if and only if $\left\{e_{j}\right\}_{j=1}^{N}$ and $\left\{f_{j}\right\}_{n=1}^{N}$ are dual frames for $\mathbb{K}^{d}$.

$(1) \Leftrightarrow(4)$. Similarly, the definition of $\mathcal{A}$ shows that

$$
\left(\mathcal{A}^{2}\right)_{i k}=\sum_{j=1}^{N} \mathcal{A}_{i j} \mathcal{A}_{j k}=\sum_{j=1}^{N}\left\langle e_{i}, f_{j}\right\rangle\left\langle e_{j}, f_{k}\right\rangle=\left\langle\sum_{j=1}^{N}\left\langle e_{i}, f_{j}\right\rangle e_{j}, f_{k}\right\rangle .
$$

This gives that $\mathcal{A}^{2}=\mathcal{A}$ if and only if $\left\{e_{j}\right\}_{j=1}^{N}$ and $\left\{f_{j}\right\}_{j=1}^{N}$ are dual frames for $\mathbb{K}^{d}$. $(1) \Leftrightarrow(6)$. By linearity of frame expansions, $\left\{e_{n}\right\}_{n=1}^{N}$ and $\left\{f_{n}\right\}_{n=1}^{N}$ are dual frames for $\mathbb{K}^{d}$ if and only if

$$
\forall 1 \leq k \leq d, \quad \delta_{k}=\sum_{n=1}^{N}\left\langle\delta_{k}, f_{n}\right\rangle e_{n}=\sum_{n=1}^{N} \overline{f_{n k}} e_{n},
$$

where $\delta_{k} \in \mathbb{K}^{d}$ is the canonical vector with $j$ th entry given by the Kronecker $\delta_{j, k}$. This is equivalent to $(6)$.

Note the similarity between the condition stated in (6) and the duality principle for Gabor frames; see [15]. 


\section{DuAl SySTEM INNER PRODUCTS}

The purpose of this section is to provide a characterization of the scalar sequences $\left\{\alpha_{n}\right\}_{n=1}^{N} \subset \mathbb{K}$ for which there exists a dual pair of frames $\left\{e_{n}\right\}_{n=1}^{N}$ and $\left\{f_{n}\right\}_{n=1}^{N}$ for $\mathbb{K}^{d}$ such that $\alpha_{n}=\left\langle e_{n}, f_{n}\right\rangle$ for all $1 \leq n \leq N$. The technical proofs are collected in Section 4

We will always assume that $N>d$. In fact, for $\left\{e_{n}\right\}_{n=1}^{N}$ to be a frame for $\mathbb{K}^{d}$ it is necessary that $N \geq d$, and in the case $N=d$ it is well known that any frame $\left\{e_{n}\right\}_{n=1}^{N}$ is a basis and has a unique dual $\left\{f_{n}\right\}_{n=1}^{N}$, which automatically satisfies that $\alpha_{n}=1$ for all $n$. Interestingly, the characterization in the case of $N>d$ is significantly different:

Theorem 3.1. Given $\left\{\alpha_{n}\right\}_{n=1}^{N} \subset \mathbb{K}$ and $N>d$ the following are equivalent:

(1) There exist dual frames $\left\{e_{n}\right\}_{n=1}^{N}$ and $\left\{f_{n}\right\}_{n=1}^{N}$ for $\mathbb{K}^{d}$ such that $\alpha_{n}=$ $\left\langle e_{n}, f_{n}\right\rangle$ for all $1 \leq n \leq N$.

(2) There exists a tight frame $\left\{g_{n}\right\}_{n=1}^{N}$ and a corresponding dual frame $\left\{h_{n}\right\}_{n=1}^{N}$ for $\mathbb{K}^{d}$ such that $\alpha_{n}=\left\langle g_{n}, h_{n}\right\rangle$ for all $1 \leq n \leq N$.

(3) $d=\sum_{n=1}^{N} \alpha_{n}$.

Motivated by Theorem 3.1 we will say that the full range for the parameters $\left\{\alpha_{n}\right\}_{n=1}^{N}$ is given by the constraint $d=\sum_{n=1}^{N} \alpha_{n}$. Surprisingly, it turns out that for each $N>d$ we can find a single frame $\left\{e_{n}\right\}_{n=1}^{N}$ such that all values in the full range can be attained as inner products $\alpha_{n}=\left\langle e_{n}, f_{n}\right\rangle$ for suitable chosen dual frames $\left\{f_{n}\right\}_{n=1}^{N}$. This motivates the following definition (see Section 4.2 for equivalent formulations).

Definition 3.2. A frame $\left\{e_{n}\right\}_{n=1}^{N} \subset \mathbb{K}^{d}$ has the full range property (FRP) if for every $\left\{\alpha_{n}\right\}_{n=1}^{N} \subset \mathbb{K}$ satisfying $\sum_{n=1}^{N} \alpha_{n}=d$ there exists a dual frame $\left\{f_{n}\right\}_{n=1}^{N} \subset \mathbb{K}^{d}$ such that $\left\langle e_{n}, f_{n}\right\rangle=\alpha_{n}$ for all $1 \leq n \leq N$.

We next aim at a characterization of the FRP. In particular our approach will give us a particularly simple choice of a frame with the FRP, and this provides us with a constructive proof of the implication $(3) \Rightarrow(1)$ in Theorem 3.1 . The next lemma will be helpful.

Lemma 3.3. Let $\left\{e_{n}\right\}_{n=1}^{N}$ be a frame for $\mathbb{K}^{d}$ and let $T$ be a $d \times d$ invertible matrix with entries in $\mathbb{K}$. Then the frame $\left\{e_{n}\right\}_{n=1}^{N}$ has the FRP if and only if the frame $\left\{T e_{n}\right\}_{n=1}^{N}$ has the FRP.

Lemma 3.3 is a useful tool for simplifying certain FRP questions. If $\left\{e_{n}\right\}_{n=1}^{N}$ is a frame for $\mathbb{K}^{d}$, then there exists a $d \times d$ invertible matrix $T$ such that $\left\{T e_{n}\right\}_{n=1}^{N}$ contains an orthonormal basis. Since $\left\{e_{n}\right\}_{n=1}^{N}$ has the FRP if and only if $\left\{T e_{n}\right\}_{n=1}^{N}$ has the FRP, it suffices to characterize certain properties of FRP for frames that contain an orthonormal basis. In view of this, Theorem 3.4 below gives a characterization of the FRP for frames containing an orthonormal basis (and hence a full characterization of the FRP up to invertible transformations).

Given $\left\{w_{j}\right\}_{j=1}^{L} \subset \mathbb{K}^{d}$ define the $(L+d) \times d$ matrices

$$
M\left(w_{j}\right)=\left(\begin{array}{c}
W_{j} \\
R_{j}
\end{array}\right)
$$

where $W_{j}$ is the $d \times d$ diagonal matrix with $\overline{w_{j}}$ on the main diagonal and $R_{j}$ is the $L \times d$ matrix that has $-\overline{w_{j}}$ as its $j$ th row and 0 everywhere else. Using $w_{j}(k)$ 
to denote the $k$ th entry of the vector $w_{j}$, the $(L+d) \times d$ matrix $M\left(w_{j}\right)$ has the following appearance:

$$
M\left(w_{j}\right)=\left(\begin{array}{ccccc}
\overline{w_{j}(1)} & 0 & 0 & \ldots & 0 \\
0 & \overline{w_{j}(2)} & 0 & \ldots & 0 \\
& & \ddots & & \\
0 & 0 & 0 & \ldots & \overline{w_{j}(d)} \\
0 & 0 & 0 & \ldots & 0 \\
-\overline{w_{j}(1)} & -\overline{w_{j}(2)} & -\overline{w_{j}(3)} & \ldots & -\overline{w_{j}(d)} \\
0 & 0 & 0 & \ldots & 0
\end{array}\right),
$$

where the row containing $-\overline{w_{j}}$ is the $(d+j)$ th row.

Theorem 3.4. Let $N>d$ and $L=N-d$. Let $\left\{e_{n}\right\}_{n=1}^{d+L}$ be a frame for $\mathbb{K}^{d}$ with the property that $\left\{e_{n}\right\}_{n=1}^{d}$ is the canonical basis for $\mathbb{K}^{d}$. Let $M_{j}=M\left(e_{d+j}\right)$, with $1 \leq j \leq L$, be the matrices associated to $\left\{e_{d+j}\right\}_{j=1}^{L}$ by (3.1), and define the $(d+L) \times(L d)$ matrix $M=\left[M_{1}, M_{2}, \ldots, M_{L}\right]$. The following are equivalent:

(1) $\left\{e_{n}\right\}_{n=1}^{N}$ has the FRP.

(2) The columns of $M$ span the vector space $H=\left\{\alpha \in \mathbb{K}^{d+L}: \sum_{n=1}^{d+L} \alpha_{n}=0\right\}$.

(3) The matrix $M$ has $\operatorname{rank}(M)=(d+L-1)$.

The following results will be derived as consequences of Theorem 3.4 and at the end lead to a simple construction of a frame with the FRP.

Proposition 3.5. Let $L \geq 1$. If the frame $\left\{e_{n}\right\}_{n=1}^{d+L}$ for $\mathbb{K}^{d}$ has the FRP and if $e_{d+L+1} \in \mathbb{K}^{d}$ is nonzero, then $\left\{e_{n}\right\}_{n=1}^{d+L+1}$ has the FRP.

For the case of a frame that just contains a single extra element (compared to the dimension of the space) the characterization simplifies:

Proposition 3.6. Let $\left\{e_{n}\right\}_{n=1}^{d+1}$ be a frame for $\mathbb{K}^{d}$. The following are equivalent:

(1) $\left\{e_{n}\right\}_{n=1}^{d+1}$ has the FRP.

(2) $\forall 1 \leq k \leq(d+1), \quad e_{k} \in \operatorname{span}\left\{e_{n}\right\}_{n \neq k}$.

Condition (2) of Proposition 3.6 is equivalent to having that any $d$-element subset of the frame $\left\{e_{n}\right\}_{n=1}^{d+1}$ is linearly independent. The next corollary follows by combining Propositions 3.5 and 3.6.

Corollary 3.7. Let $\left\{e_{n}\right\}_{n=1}^{N}$ be a frame for $\mathbb{K}^{d}$ with $N>d$. Assume that each $e_{n}$ is nonzero and assume that $\left\{e_{n}\right\}_{n=1}^{N}$ contains a subset $\left\{e_{n_{j}}\right\}_{j=1}^{d+1}$ satisfying

(1) $\operatorname{span}\left\{e_{n_{j}}\right\}_{j=1}^{d+1}=\mathbb{K}^{d}$,

(2) $\forall 1 \leq k \leq(d+1), \quad e_{n_{k}} \in \operatorname{span}\left\{e_{n_{j}}: j \neq k\right\}$

Then $\left\{e_{n}\right\}_{n=1}^{N}$ has the FRP.

The hypotheses of Corollary 3.7 are equivalent to having that all $N$ of the frame vectors $\left\{e_{n}\right\}_{n=1}^{N}$ are nonzero and that there is a subset of $(d+1)$ frame vectors with the property that any $d$ frame vectors of this subset are linearly independent. Finally, by combining the obtained results we arrive at the following simple and concrete example of a frame having the FRP. 
Example 3.8. Let $\left\{e_{n}\right\}_{n=1}^{d}$ be the canonical basis for $\mathbb{K}^{d}$ and define $e_{d+1}=$ $\sum_{n=1}^{d} e_{n}$. By Proposition 3.6 $\left\{e_{n}\right\}_{n=1}^{d+1}$ has the FRP. Moreover, by Corollary 3.7. if $\left\{e_{n}\right\}_{n=d+2}^{N} \subset \mathbb{K}^{d}$ are arbitrary and nonzero, then $\left\{e_{n}\right\}_{n=1}^{N}$ has the FRP.

Even with the above results and examples in mind, it is unclear how restrictive the FRP is. The next example shows that in a probabilistic sense almost all frames have the FRP:

Example 3.9. Define the $d \times N$ synthesis operator $E$ by independently drawing its entries from the normal distribution $N(0,1)$. Let $\left\{e_{n}\right\}_{n=1}^{N} \subset \mathbb{R}^{d}$ be the columns of $E$. It follows from Corollary 3.7 and standard properties of Gaussian random vectors that, with probability one, $\left\{e_{n}\right\}_{n=1}^{N}$ is a frame for $\mathbb{K}^{d}$ and has FRP.

\section{Proofs of the Results in Section 3}

4.1. Proof of Theorem 3.1. (1) $\Rightarrow(2)$. Fix $\alpha=\left\{\alpha_{n}\right\}_{n=1}^{N} \subset \mathbb{K}$ such that there exists a dual frame pair $\left\{e_{n}\right\}_{n=1}^{N},\left\{f_{n}\right\}_{n=1}^{N} \subset \mathbb{K}^{d}$ satisfying $\alpha_{n}=\left\langle e_{n}, f_{n}\right\rangle$. Note that if $E, F$ are the synthesis operators for $\left\{e_{n}\right\}_{n=1}^{N},\left\{f_{n}\right\}_{n=1}^{N}$, then

$$
F E^{*}=I \quad \text { and } \quad \alpha=\operatorname{diag}\left(F^{*} E\right),
$$

where $\operatorname{diag}(\cdot)$ denotes the column vector of entries on the main diagonal of a matrix.

Let $S=E E^{*}$ be the frame operator for $\left\{e_{n}\right\}_{n=1}^{N}$ and let $\left\{g_{n}\right\}_{n=1}^{N}=\left\{S^{-1 / 2} e_{n}\right\}_{n=1}^{N}$ be the associated canonical tight frame. Note that $S$ and $S^{1 / 2}$ and $S^{-1 / 2}$ are selfadjoint and that the synthesis operator associated with $\left\{g_{n}\right\}_{n=1}^{N}$ is given by

$$
G=S^{-1 / 2} E .
$$

Consider the sequence $\left\{h_{n}\right\}_{n=1}^{N} \subset \mathbb{K}^{d}$ defined by the synthesis operator $H=$ $S^{1 / 2} F$. Then

$$
H G^{*}=S^{1 / 2} F E^{*} S^{-1 / 2}=S^{1 / 2} I S^{-1 / 2}=I ;
$$

i.e., $\left\{h_{n}\right\}_{n=1}^{N}$ is a dual frame of $\left\{g_{n}\right\}_{n=1}^{N}$. Moreover, we have $H^{*} G=F^{*} S^{1 / 2} S^{-1 / 2} E$ $=F^{*} E$, which gives

$$
\operatorname{diag}\left(H^{*} G\right)=\operatorname{diag}\left(F^{*} E\right)=\alpha .
$$

Thus, as required, we have shown that $\left\{g_{n}\right\}_{n=1}^{N} \subset \mathbb{K}^{d}$ is a tight frame with dual frame $\left\{h_{n}\right\}_{n=1}^{N}$ and that $\alpha_{n}=\left\langle g_{n}, h_{n}\right\rangle$.

$(2) \Rightarrow(3)$. Let $G, H$ be the synthesis operators for $\left\{g_{n}\right\}_{n=1}^{N},\left\{h_{n}\right\}_{n=1}^{N}$. In terms of $G, H$ the dual frame condition reads $G H^{*}=I$. Thus $d=\operatorname{trace}\left(G H^{*}\right)=$ $\operatorname{trace}\left(H^{*} G\right)=\sum_{n=1}^{N}\left\langle g_{n}, h_{n}\right\rangle$.

$(3) \Rightarrow(1)$. As already stated this will be a consequence of Example 3.8

4.2. The full range property and Lemma 3.3. We begin with a few preliminary remarks before we prove the results concerning the full range property.

The equivalence $(1) \Leftrightarrow(2)$ of Proposition 2.1 and the implication $(1) \Rightarrow(3)$ proven in Proposition 3.1 (now applied to the canonical dual frame $f_{n}=\widetilde{e}_{n}$ ) give the following equivalent formulation of the FRP. The frame $\left\{e_{n}\right\}_{n=1}^{N} \subset \mathbb{K}^{d}$ has the 
FRP if and only if for every $\left\{\alpha_{n}\right\}_{n=1}^{N} \subset \mathbb{K}$ satisfying $\sum_{n=1}^{N} \alpha_{n}=0$ there exists $\left\{z_{n}\right\}_{n=1}^{N} \subset \mathbb{K}^{d}$ so that

$$
\forall x \in \mathbb{R}^{d}, \quad 0=\sum_{n=1}^{N}\left\langle x, e_{n}\right\rangle z_{n}=\sum_{n=1}^{N}\left\langle x, z_{n}\right\rangle e_{n}
$$

and such that $\left\langle e_{n}, z_{n}\right\rangle=\alpha_{n}$ for all $1 \leq n \leq N$.

For our purposes it will be useful to equivalently formulate the FRP directly in terms of the synthesis operators $E, Z$ associated to $\left\{e_{n}\right\}_{n=1}^{N},\left\{z_{n}\right\}_{n=1}^{N}$. The frame $\left\{e_{n}\right\}_{n=1}^{N}$ has the FRP if and only if for every $\alpha=\left[\alpha_{1}, \alpha_{2}, \ldots, \alpha_{N}\right]^{T} \in \mathbb{K}^{d}$ satisfying $\sum_{n=1}^{N} \alpha_{n}=0$ there exists $Z$ satisfying $Z E^{*}=E Z^{*}=0$ and $\alpha=\operatorname{diag}\left(Z^{*} E\right)$.

Proof of Lemma 3.3. It suffices to show that if $\left\{e_{n}\right\}_{n=1}^{N}$ has the FRP, then $\left\{T e_{n}\right\}_{n=1}^{N}$ has the FRP. Since $T$ is invertible,

$$
\left(\forall x \in \mathbb{K}^{d}, \quad 0=\sum_{n=1}^{N}\left\langle x, e_{n}\right\rangle z_{n}\right) \Longleftrightarrow\left(\forall x \in \mathbb{K}^{d}, \quad 0=\sum_{n=1}^{N}\left\langle x, T e_{n}\right\rangle\left(T^{*}\right)^{-1} z_{n}\right) .
$$

The proof now follows from $\left\langle e_{n}, z_{n}\right\rangle=\left\langle T e_{n},\left(T^{*}\right)^{-1} z_{n}\right\rangle$.

4.3. Proof of Theorem 3.4. Step I. Let $E$ be the $d \times(d+L)$ synthesis operator associated to $\left\{e_{n}\right\}_{n=1}^{N}$. Since $E$ has full rank, the rows of $E$ span a $d$-dimensional subspace $S \subset \mathbb{K}^{d+L}$. Note that the first $d$ columns of $E$ form a $d \times d$ identity matrix. Thus the row vectors $\left\{v_{j}\right\}_{j=1}^{L} \subset \mathbb{K}^{d+L}$ defined by

$$
v_{j}=\left[\overline{e_{d+j}},-\delta_{j}\right]=\left[\overline{e_{d+j}(1)}, \overline{e_{d+j}(2)}, \ldots, \overline{e_{d+j}(d)}, 0, \ldots,-1, \ldots, 0\right]
$$

span the $L$-dimensional subspace $S^{\perp}$. Here $\delta_{j} \in \mathbb{K}^{L}$ is defined by $\delta_{j}(k)=\delta_{j, k}$.

Step II. Note that the synthesis operator $Z$ associated to $\left\{z_{n}\right\}_{n=1}^{d+L} \subset \mathbb{K}^{d}$ satisfies $0=Z E^{*}=E Z^{*}$ if and only if each row of $Z$ is a linear combination of the vectors $\left\{v_{j}\right\}_{j=1}^{L}$ from (4.2). In other words, for each $1 \leq k \leq d$, the $k$ th row of $Z$ has the form $\sum_{j=1}^{L} c_{j}(k) v_{j}$ for some constants $c_{1}(k), c_{2}(k), \ldots, c_{L}(k) \in \mathbb{K}$. This in turn is equivalent to having that

$$
\forall 1 \leq n \leq(d+L), \quad z_{n}=n \text {th column of } Z=\sum_{j=1}^{L} v_{j}(n) c_{j},
$$

where $c_{j}=\left[c_{j}(1), c_{j}(2), \ldots, c_{j}(d)\right]^{T}$. In summary, $Z E^{*}=E Z^{*}=0$ if and only if (4.3) holds for some vectors $\left\{c_{j}\right\}_{j=1}^{L} \subset \mathbb{K}^{d}$.

Step III. Suppose that $\left\{z_{n}\right\}_{n=1}^{d+L} \subset \mathbb{K}^{d}$ has the synthesis operator $Z$ satisfying $Z E^{*}=$ $E Z^{*}=0$. By (4.3), we have for $1 \leq k \leq(d+L)$,

$$
\alpha_{k}=\left\langle e_{k}, z_{k}\right\rangle=\sum_{j=1}^{L} \overline{v_{j}(k)}\left\langle e_{k}, c_{j}\right\rangle=\sum_{j=1}^{L} \overline{v_{j}(k)\left\langle c_{j}, e_{k}\right\rangle} .
$$

In other words, $\alpha=\sum_{j=1}^{L} \overline{D_{j} E^{*} c_{j}}$, where $D_{j}$ is the diagonal matrix with $v_{j}$ on its main diagonal. Recalling that $\left\{e_{n}\right\}_{n=1}^{d}$ is the canonical basis, the structure of 
$E, M_{j}$ and $v_{j}$ gives that $M_{j}=D_{j} E^{*}$, which yields

$$
\alpha=\sum_{j=1}^{L} \overline{M_{j} c_{j}} .
$$

Step $I V$. We now show that $(1) \Rightarrow(2)$. Suppose that $\left\{e_{n}\right\}_{n=1}^{d+L}$ has the FRP. Note that the columns of $M$ are in $H=\left\{\alpha \in \mathbb{R}^{d+L}: \sum_{n=1}^{d+L} \alpha_{n}=0\right\}$ by construction. Since $E$ has the FRP, it follows from (4.5) that the columns of $M=\left[M_{1}, M_{2}, \ldots, M_{L}\right]$ must span $H$.

Step $V$. We now show that $(2) \Rightarrow(1)$. Note that $H$ has dimension $(d+L-1)$ and each column of $M$ is in $H$. By hypothesis, given any $\alpha=\left[\alpha_{1}, \ldots, \alpha_{d+L}\right]^{T} \in \mathbb{K}^{d+L}$ satisfying $0=\sum_{n=1}^{d+L} \alpha_{n}$ there exist vectors $\left\{c_{j}\right\}_{j=1}^{L} \subset \mathbb{K}^{d}$ such that $\alpha=\sum_{j=1}^{L} \overline{M_{j} c_{j}}$. Now define $Z$ from $\left\{c_{j}\right\}_{j=1}^{L}$ and $\left\{v_{j}\right\}_{j=1}^{L}$ using (4.3). It follows that $Z^{*} E=E^{*} Z=0$. Moreover, by (4.4) and (4.5) we have $\alpha_{n}=\left\langle e_{n}, z_{n}\right\rangle$. Thus $\left\{e_{n}\right\}_{n=1}^{d+L}$ has FRP.

Step VI. Finally, $(2) \Leftrightarrow(3)$ is clear since $H$ has dimension $(d+L-1)$ and since each column of $M$ is in $H$.

4.4. Proof of Proposition 3.5. Let $T$ be an invertible matrix for which $\left\{T e_{n}\right\}_{n=1}^{N}$ is the canonical basis for $\mathbb{K}^{d}$. By Lemma 3.3. $\left\{e_{n}\right\}_{n=1}^{N}$ has FRP if and only if $\left\{T e_{n}\right\}_{n=1}^{N}$ has FRP. Thus for the proof one may assume without loss of generality that $\left\{e_{n}\right\}_{n=1}^{d}$ is the canonical basis for $\mathbb{K}^{d}$.

Let $\left\{M_{j}\right\}_{j=1}^{L}$ be the $(L+d) \times d$ matrices associated to $\left\{e_{n}\right\}_{n=d+1}^{d+L}$ by (3.1). Similarly, let $\left\{M_{j}^{\prime}\right\}_{j=1}^{L+1}$ be the $(L+d+1) \times d$ matrices associated to $\left\{e_{n}\right\}_{n=d+1}^{d+L+1}$ by (3.1). Note that for $1 \leq j \leq L$,

$$
M_{j}^{\prime}=\left(\begin{array}{c}
M_{j} \\
0_{1 \times d}
\end{array}\right) .
$$

Let $M=\left[M_{1}, \ldots, M_{L}\right]$ and $M^{\prime}=\left[M_{1}^{\prime}, \ldots, M_{L}^{\prime}, M_{L+1}^{\prime}\right]$. By Theorem 3.4 the columns of $M$ span the vector space $H=\left\{\alpha \in \mathbb{K}^{d+L}: \sum_{n=1}^{d+L} \alpha_{n}=0\right\}$. Therefore, the columns of $M_{1}^{\prime}, \ldots, M_{L}^{\prime}$ span the vector space $H_{0}^{\prime}=\left\{\alpha \in \mathbb{K}^{d+L+1}\right.$ : $\alpha_{d+L+1}=0 \quad$ and $\left.\quad \sum_{n=1}^{d+L} \alpha_{n}=0\right\}$. Since $e_{d+L+1} \neq 0$ at least one column of $M_{L+1}^{\prime}$ is nontrivial; since this column is in $H^{\prime}=\left\{\alpha \in \mathbb{K}^{d+L+1}: \sum_{n=1}^{d+L} \alpha_{n}=0\right\}$ but is not in $H_{0}$, it follows that the columns of $M^{\prime}$ span $H^{\prime}$. Hence $\left\{e_{n}\right\}_{n=1}^{d+L+1}$ has the FRP.

4.5. Proof of Proposition [3.6. We may assume that $e_{n} \neq 0$ for all $1 \leq n \leq$ $(d+1)$. Indeed, it is clear that if some $e_{n}=0$, then neither condition (1) nor (2) of the theorem can hold for the frame $\left\{e_{n}\right\}_{n=1}^{d+1}$.

$(1) \Rightarrow(2)$. We prove the contrapositive. Suppose that there exists $1 \leq k \leq(d+1)$ such that $e_{k} \notin \operatorname{span}\left\{e_{n}\right\}_{n \neq k}$. Let $\left\{f_{n}\right\}_{n=1}^{d+1}$ be any dual frame to $\left\{e_{n}\right\}_{n=1}^{d+1}$. Define $\varphi=e_{k}-P_{k} e_{k}$, where $P_{k}$ is the orthogonal projection onto $\operatorname{span}\left\{e_{n}\right\}_{n \neq k}$. Since $\varphi \perp\left\{e_{n}\right\}_{n \neq k}$ we have

$$
\varphi=\sum_{n=1}^{d+1}\left\langle\varphi, e_{n}\right\rangle f_{n}=\left\langle\varphi, e_{k}\right\rangle f_{k} .
$$

Since $\left\langle\varphi, e_{k}\right\rangle \neq 0$ this implies that $\left\langle f_{k}, e_{k}\right\rangle=\left\langle e_{k}, f_{k}\right\rangle=1$. So $\left\{e_{n}\right\}_{n=1}^{d+1}$ cannot have the FRP. 
$(2) \Rightarrow(1)$. Since $\left\{e_{n}\right\}_{n=1}^{d+1}$ is a frame there exists an invertible matrix $T$ such that $\left\{T e_{n}\right\}_{n=1}^{d}$ is the canonical basis for $\mathbb{K}^{d}$. Moreover, since $T$ is invertible, condition (2) still holds for the system $\left\{T e_{n}\right\}_{n=1}^{d+1}$. However, since $\left\{T e_{n}\right\}_{n=1}^{d}$ is the canonical basis, this can only be the case if all entries of $T e_{d+1}$ are nonzero, i.e., $\left(T e_{d+1}\right)(j) \neq 0$ for $1 \leq j \leq d$. In particular the synthesis operator for $\left\{T e_{n}\right\}_{n=1}^{d+1}$ has the form

$$
\Phi=\left(\begin{array}{ccccc}
1 & 0 & \cdots & 0 & x_{1} \\
0 & 1 & \cdots & 0 & x_{2} \\
& & \ddots & & \\
0 & 0 & \cdots & 1 & x_{d}
\end{array}\right),
$$

where $x_{1}, x_{2}, \ldots, x_{d} \neq 0$. Since the system $\left\{z_{n}\right\}_{n=1}^{d+1} \subset \mathbb{K}^{d}$ with analysis operator

$$
Z^{*}=\left(\begin{array}{cccc}
\alpha_{1} & \frac{\alpha_{1} x_{1}}{x_{2}} & \ldots & \frac{\alpha_{1} x_{1}}{x_{d}} \\
\frac{\alpha_{1} x_{2}}{x_{1}} & \alpha_{2} & \ldots & \frac{\alpha_{1} x_{2}}{x_{d}} \\
& & \ddots & \\
\frac{\alpha_{1} x_{d}}{x_{1}} & \frac{\alpha_{1} x_{d}}{x_{2}} & \ldots & \alpha_{d} \\
-\frac{\alpha_{1}}{x_{1}} & -\frac{\alpha_{2}}{x_{2}} & \cdots & -\frac{\alpha_{d}}{x_{d}}
\end{array}\right)
$$

satisfies $\Phi Z^{*}=Z \Phi^{*}=0$ and $\operatorname{diag}\left(Z^{*} \Phi\right)=\left[\alpha_{1}, \ldots, \alpha_{d},-\sum_{n=1}^{d} \alpha_{n}\right]^{T}$ it follows that $\left\{T e_{n}\right\}_{n=1}^{d+1}$ has the FRP. Thus by Proposition 3.3, $\left\{e_{n}\right\}_{n=1}^{d+1}$ has the FRP.

\section{ACKNOWLEDGEMENTS}

The second author was partly supported by NSF DMS Grant 0811086. This author thanks the Academia Sinica Institute of Mathematics (Taipei, Taiwan) and the Technical University of Denmark (Lyngby, Denmark) for their hospitality and support.

The third author thanks the Department of Mathematics at the Technical University of Denmark for hospitality and support during a visit from October 2009 to April 2010. He also acknowledges support by the third innovative talent training of 211 project (2009), Xiamen University.

\section{REFERENCES}

[1] J. J. Benedetto, A. Powell and Ö. Yilmaz, Sigma-Delta quantization and finite frames, IEEE Trans. Inform. Theory 52 (2006), 1990-2005. MR2234460 (2007a:94030)

[2] J. J. Benedetto and M. Fickus, Finite normalized tight frames, Adv. Comput. Math. 18 (2003), 357-385. MR1968126(2004c:42059)

[3] J. Blum, M. Lammers, A.M. Powell and Ö. Yılmaz, Sobolev duals in frame theory and SigmaDelta quantization, J. Fourier Anal. Appl. 16 (2010), 365-382. MR2643587 (2011e:42062)

[4] P.G. Casazza, M. Fickus, J. Kovačević, M. Leon and J.C. Tremain, A physical interpretation of tight frames. In: Harmonic analysis and applications, 51-76, Appl. Numer. Harmon. Anal., Birkhäuser Boston, Boston, MA, 2006. MR2249305 (2007d:42053)

[5] P.G. Casazza, Custom building finite frames. Contemporary Mathematics 345, Amer. Math. Soc. (2004), 61-86. MR2066822 (2005f:42078)

[6] P.G. Casazza and M. Leon, Frames with a given frame operator. Preprint. http://www.math. missouri.edu/ pete/pdf/PM2.pdf

[7] P.G. Casazza and N. Leonhard, Classes of finite equal norm Parseval frames. Contemporary Mathematics 451, Amer. Math. Soc. (2008), 11-31. MR2422239 (2009f:42032)

[8] P.G. Casazza and J. Kovačević, Equal-norm tight frames with erasures. Adv. Comput. Math. 18 (2003), 387-430. MR1968127(2004e:42046) 
[9] O. Christensen, An introduction to frames and Riesz bases, Birkhäuser Boston, Boston, MA, 2003. MR 1946982 (2003k:42001)

[10] V. Goyal, J. Kovačević and J. Kelner, Quantized frame expansions with erasures, Appl. Comput. Harmon. Anal. 10 (2001), 203-233. MR.1829801(2002h:94012)

[11] M. Lammers and A. Maeser, An uncertainty principle for finite frames, J. Math. Anal. Appl. 373 (2011), 242-247. MR2684474 (2011g:42079)

[12] M. Lammers, A.M. Powell and O. Yilmaz, Alternative dual frames for digital-to-analog conversion in sigma-delta quantization, Adv. Comput. Math. 32 (2010), 73-102. MR.2574568 (2010m:42073)

[13] C.S. Güntürk, M. Lammers, A.M. Powell, R. Saab and Ö. Yılmaz, Sobolev duals for random frames and $\Sigma \Delta$ quantization of compressed sensing measurements. Preprint, 2010.

[14] S. Li, On general frame decompositions, Numer. Funct. Anal. Optim. 16 (1995), 1181-1191. MR.1374971 (97b:42055)

[15] A. Ron and Z. Shen, Weyl-Heisenberg systems and Riesz bases in $L^{2}\left(\mathbb{R}^{d}\right)$, Duke Math. J. 89 (1997), 237-282. MR 1460623 (98i:42013)

Department of Mathematics, Technical University of Denmark, Building 303, 2800 LYNGBY, DENMARK

E-mail address: 0le.Christensen@mat.dtu.dk

Department of Mathematics, Vanderbilt University, Nashville, Tennessee 37240

E-mail address: alexander.m.powell@vanderbilt.edu

Department of Mathematics, Xiamen University, Xiamen 361005, People's Republic OF CHINA

E-mail address: xxc570@163.com 